Brazilian Journal of Medical and Biological Research (2008) 41: 437-438

ISSN 0100-879X

\title{
Retraction
}

\section{Retraction of the paper "Niemann-Pick type C1 protein influences the delivery of cholesterol to the SREBP:SCAP complex"}

Yong-Jun Guo, Wei-Hua Li, Rong Wu, Qiang Xie, Zu-Huang Zhang and Lian-Qun Cui Braz J Med Biol Res 2008; 41: 26-33

The Editors of the Brazilian Journal of Medical and Biological Research are retracting the paper "Niemann-Pick type C1 protein influences the delivery of cholesterol to the SREBP:SCAP complex". Yong-Jun Guo, Wei-Hua Li, Rong Wu, Qiang Xie, Zu-Huang Zhang, Lian-Qun Cui. Braz J Med Biol Res 2008; 41: 26-33 because of plagiarism. The person responsible, Y.J. Guo, Department of Cardiology, Shandong Provincial Hospital, Fujian Medical University, Xiamen, China, has admitted this breach of ethics, has stated that the other authors were not aware of the plagiarism, and sends his sincere apologies to all involved. The original work was published in an Honour Thesis by Julie Wood of the University of New South Wales, Sydney, Australia. 